REVISTA PORTUGUESA DE PSICOLOGA

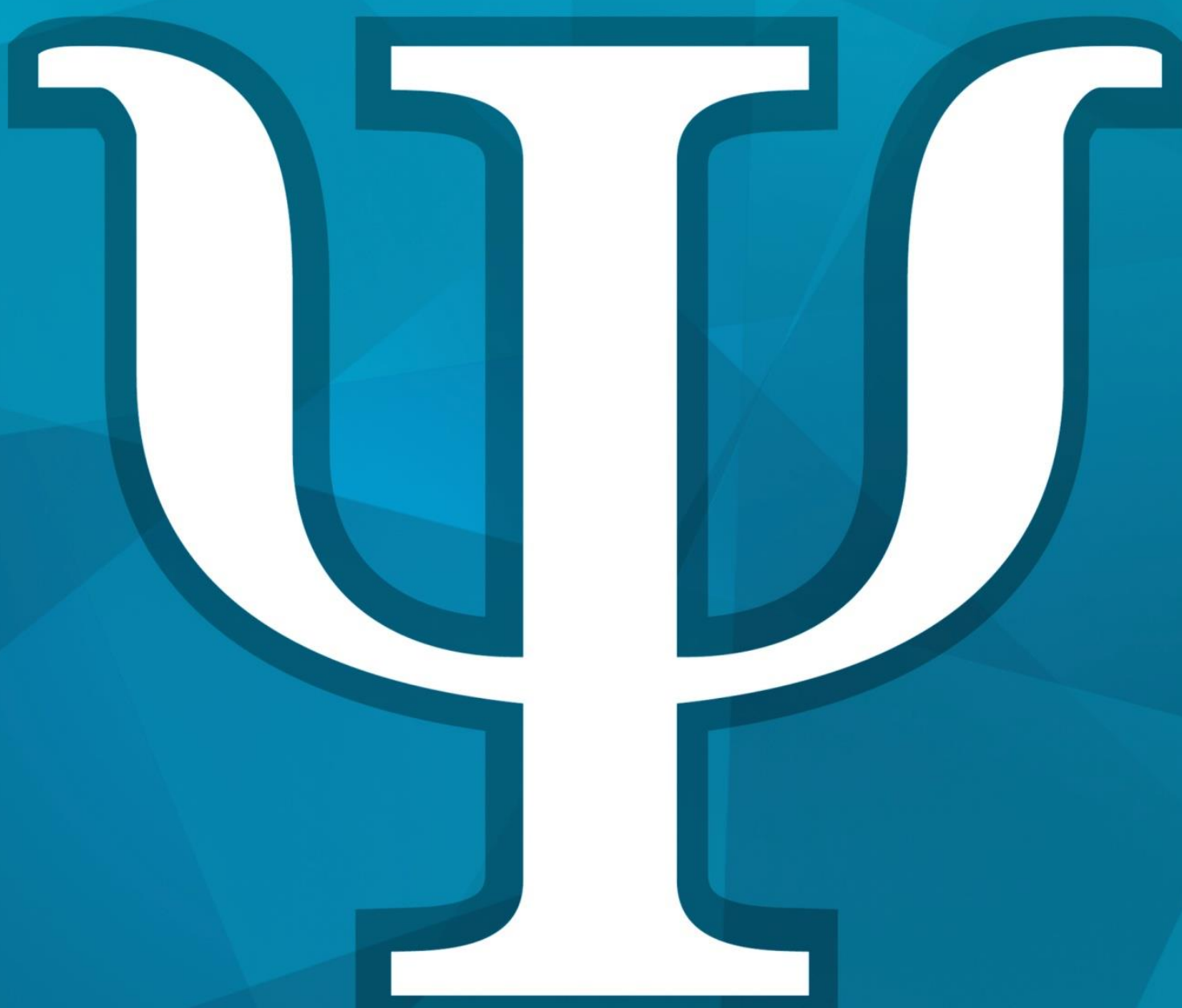




\section{Conselho Editorial / Editorial Board}

\section{Editor:}

João Manuel Moreira

Faculdade de Psicologia, Universidade de Lisboa

\section{Conselho Editorial / Editorial Board:}

Adelina Lopes da Silva

Faculdade de Psicologia, Universidade de Lisboa

Alexandra Reis

Faculdade de Ciências Humanas e Sociais, Universidade do Algarve

Amâncio da Costa Pinto

Faculdade de Psicologia e CE, Universidade do Porto

Ana Margarida Veiga Simão

Faculdade de Psicologia, Universidade de Lisboa

Bárbara Figueiredo

Escola de Psicologia, Universidade do Minho

Danilo Silva

Faculdade de Psicologia, Universidade de Lisboa

Félix Neto

Faculdade de Psicologia e CE, Universidade do Porto

Helio Carpinteiro

Universidade Complutense de Madrid

Isabel Sá

Faculdade de Psicologia, Universidade de Lisboa

Isabel Soares

Escola de Psicologia, Universidade do Minho

Jean Guichard

Conservatoire National des Artes et Métiers

José H. Ferreira-Marques

Faculdade de Psicologia, Universidade de Lisboa

José Keating

Escola de Psicologia, Universidade do Minho

José Tomás da Silva

Faculdade de Psicologia e CE, Universidade de Coimbra

Leonel Garcia-Marques

Faculdade de Psicologia, Universidade de Lisboa

Leonor Cardoso

Faculdade de Psicologia e CE, Universidade de Coimbra

Luísa Barros

Faculdade de Psicologia, Universidade de Lisboa

Luísa Morgado

Faculdade de Psicologia e CE, Universidade de Coimbra

Manuel Rafael

Faculdade de Psicologia, Universidade de Lisboa

Maria do Céu Taveira

Escola de Psicologia, Universidade do Minho

\author{
Maria Eduarda Duarte \\ Faculdade de Psicologia, Universidade de Lisboa \\ Maria José Chambel \\ Faculdade de Psicologia, Universidade de Lisboa
}

Mário Ferreira

Faculdade de Psicologia, Universidade de Lisboa

Mário Simões

Faculdade de Psicologia e CE, Universidade de Coimbra

Mark Savickas

Northeast Ohio Medical University

Paulo Ventura

Faculdade de Psicologia, Universidade de Lisboa

Pedro B. Albuquerque

Escola de Psicologia, Universidade do Minho

Rita Francisco

Fac. de Ciências Humanas, Univ. Católica Portuguesa

Rocío Fernandez-Ballesteros

Universidad Autónoma de Madrid

Rosário Lima

Faculdade de Psicologia, Universidade de Lisboa

Salomé Vieira Santos

Faculdade de Psicologia, Universidade de Lisboa

São Luís Castro

Faculdade de Psicologia e CE, Universidade do Porto

\section{Assistente Editorial / Editorial Assistant:}

Rita Monteiro / Inês Dias

Editores e Directores Eméritos / Emeriti Editors and Directors:

Henrique Barahona Fernandes

Director, 1967-1991

Agostinho Pereira

Editor, 1967-1969

José Luiz Simões da Fonseca Editor, 1969-1971

José H. Ferreira-Marques

1992-2001

Danilo Silva

2002-2005

Manuel Rafael

2006-2011

\section{Instruções aos autores:}

https://sites.google.com/site/revistaportuguesadepsicologia /instrucoes-aos-autores

ISSN 2183-3699 (versão electrónica / electronic version) ISSN 0872-0304 (versão impressa / print version) 


\title{
Tomada de decisão de profissionais sobre a retirada de menores à família em situações de maus-tratos físicos
}

\author{
Ana Santos \\ Escola de Psicologia, Universidade do Minho \\ João Lopes \\ Escola de Psicologia, Universidade do Minho
}

\begin{abstract}
RESUMO
O presente estudo tem como objetivo compreender de que forma grupos de profissionais (psicólogos, professores, educadores sociais e assistentes sociais) ponderam uma eventual retirada de crianças e jovens em risco à família. A amostra é constituída por 68 participantes que responderam a um questionário online. Após a apresentação de casos de maus-tratos físicos em formato de vinheta, foi solicitado que respondessem a um conjunto de questões sobre: (a) os profissionais que envolveriam na tomada de decisão, (b) os serviços que recomendariam e (c) a importância atribuída a um conjunto de características específicas de situações de maus-tratos. Foram ainda desenvolvidas análises para compreender o papel preditivo da profissão e local de trabalho dos participantes nas tomadas de decisão. Os resultados mostraram associações significativas entre diversas variáveis em estudo. Características como a gravidade, duração e recorrência do abuso foram consideradas muito importantes pelos participantes, ao contrário do nível socioeconómico das famílias.
\end{abstract}

Palavras-chave: maus-tratos; tomada de decisão; maus-tratos físicos; gravidade do abuso; retirada de menores

\begin{abstract}
This study focused on understanding how groups of professionals (psychologists, teachers, social educators and social workers) think about a possible removal of children from their families. A sample of 68 participants answered an online questionnaire. A number of physical abuse cases were presented in a vignette format and then participants were asked a set of questions about: i) professionals they think should be involved in decision making, ii) services they would recommend and iii) the importance they attribute to a set of characteristics specific to abuse situations. A study about the predictive power of professionals' affiliation and professionals' was also conducted. Results showed significant associations between several variables in study. Features such as severity, duration and recurrence of abuse were considered very important by participants, unlike families SES.
\end{abstract}

Keywords: child maltreatment; decision-making; physical abuse; severity of abuse; out-of-home placement

\section{INTRODUÇÃO}

\section{O Processo de Tomada de Decisão}

As tomadas de decisão sobre situações de maus-tratos a crianças e jovens constituem o quotidiano dos serviços sociais. Porém, em muito casos, as decisões podem carecer de uma base de informação sólida e concreta (Gambrill, 2011). Neste processo surgem, com frequência, conflitos, pelo que as incertezas e hesitações parecem bastante mais prováveis do que as tomadas de decisão seguras (Benbenishty, Osmo, \& Gold, 2003; Cleaver \& Walker, 2004). 
Numa situação ideal, a qualidade da decisão seria ponderada a partir dos melhores resultados possíveis para os menores e para a sua família (Yoo \& Brooks, 2005). No entanto, independentemente da qualidade da decisão tomada pelos profissionais, é provável que do processo resultem algumas consequências negativas para as vítimas (Munro, 2010).

A tomada de decisão específica em situações de maus-tratos a menores tem como objetivo, através do estabelecimento de limites, a proteção das crianças e jovens relativamente a quaisquer maus-tratos de que possam estar a ser alvo (DePanfilis \& Girvin, 2005; DeRoma, Kessler, McDaniel, \& Soto, 2006; English, Marshall, Coghlan, Brummel, \& Orme, 2002). A investigação sugere a necessidade de os profissionais agirem de acordo com três diretrizes: (a) analisar as denúncias realizadas, (b) ponderar a medida mais adequada para cada situação específica e (c) selecionar os serviços apropriados para a família, tendo em vista o bem-estar do menor (Crea, 2010). É ainda importante não descurar a severidade do maltrato e o risco de recorrência (Cross \& Casanueva, 2009). Esta tomada de decisão implica, não raras vezes, a intervenção intrusiva no contexto familiar, o que tem consequências importantes não só para a vítima mas também para a sua família (Davidson-Arad \& Benbenishty, 2008; DavidsonArad, Englechin-Segal, \& Wozner, 2003; Schwalbe, 2004; Slep \& Heyman, 2006).

Em geral, a investigação refere que as decisões procuram tomar em consideração todos os contextos em que a vítima está inserida, com ponderação não só da evidência física e psicológica do maltrato, como também de informação sobre a família (Crea, 2010). Não é uma decisão linear mas antes uma decisão altamente problemática e sujeita a enviesamentos. Estes resultam, em parte, da falta de clareza de linhas legislativas orientadoras, mas também de informação por vezes incompleta e/ou incorreta quanto à ocorrência dos maus-tratos e à imprevisibilidade da sua recorrência (Benbenishty et al., 2003; Gambrill, 2005; Ibanez, Borrego, Pemberton, \& Terao, 2006). A perceção da inevitabilidade destas dificuldades e enviesamentos pode limitar a confiança dos técnicos nas suas próprias tomadas de decisão. Consequentemente, pode fazer aumentar a desconfiança do público em geral, que é ciclicamente confrontado, nos meios de comunicação social, com decisões que parecem ter-se revelado inadequadas e das quais advieram consequências trágicas (Baird \& Wagner, 2000; Everson \& Sandoval, 2011; Herman, 2005).

\section{Fatores Importantes para a Tomada de Decisão}

Existe um número considerável de fatores de risco potencialmente associados a situações de maus-tratos infantis. Estes fatores não se constituem, em si mesmos, como originadores diretos dos maus-tratos, pelo que devem ser considerados num processo avaliativo abrangente. O modelo ecológico desenvolvido por Bronfenbrenner (Brofenbrenner, 1977) tem constituído um referencial na avaliação das problemáticas infantis por parte de profissionais, incluindo as situações de maus-tratos (Comissão Nacional de Proteção das Crianças e Jovens em Risco, 2011). Os Department of Health, Department of Education and Employment Home Office (2000), por exemplo, desenvolveram um modelo denominado Modelo Ecológico de Avaliação e Intervenção em Situações de Risco e de Perigo, adaptado do modelo original de Bronfenbrenner (Figura 1). 


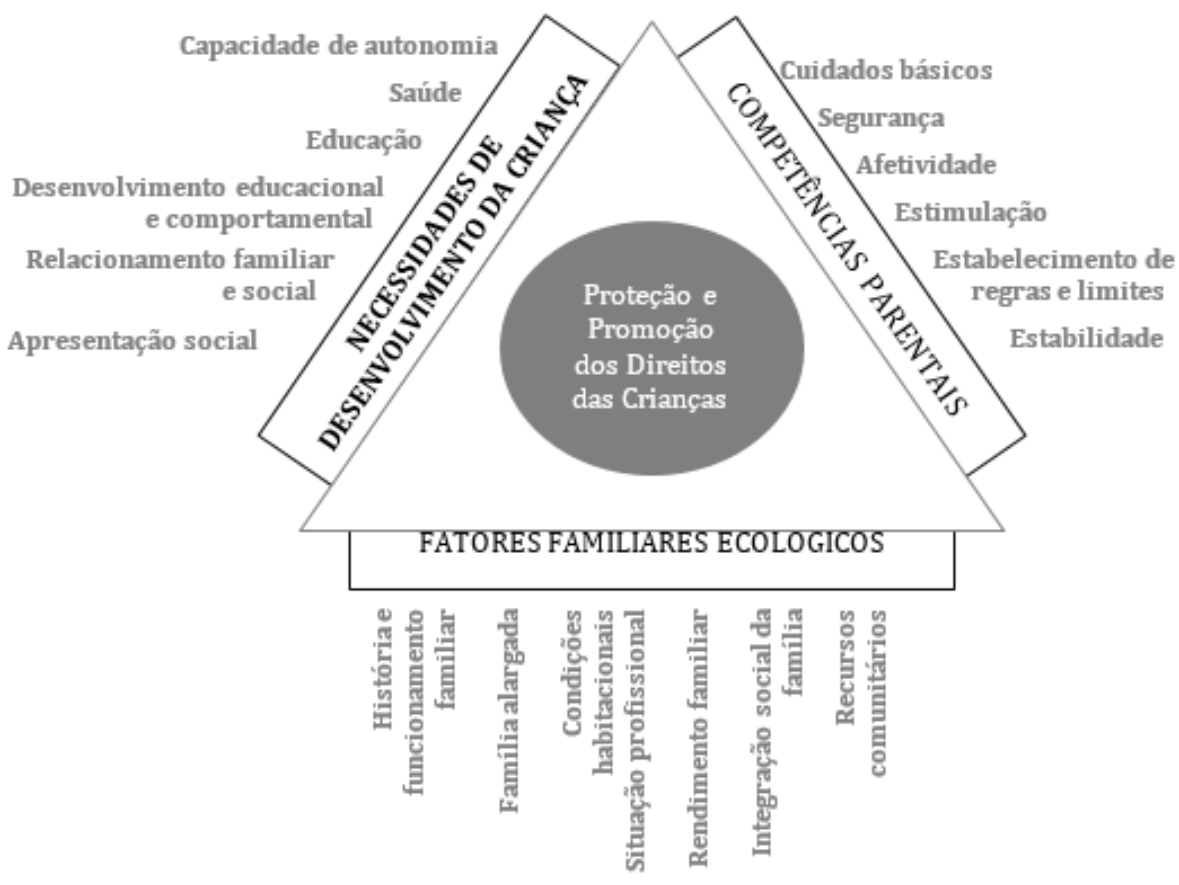

Figura 1. Modelo Ecológico de Avaliação e Intervenção em Situações de Risco e de Perigo adaptado de Department of Health, Department for Education and Employment, Home Office (2000).

O processo de tomada de decisão é complexo e não assenta apenas em conhecimentos profissionais claros ou linhas orientadoras explícitas (Drury-Hudson, 1999). Fatores pessoais e situacionais podem influenciar a posição de cada profissional face a uma situação específica de maus-tratos (Wells, Lyons, Doueck, Brown, \& Thomas, 2004).

Os fatores pessoais referem-se a todas as características do avaliador, que direta ou indiretamente, conscientemente ou não, podem afetar a sua decisão (Arruabarrena \& De Paúl, 2012). A subjetividade, os valores e as crenças parecem ser fatores essenciais, pois estão presentes desde logo na interpretação individual do conceito "superior interesse da criança" (Ashton, 2001; Britner \& Mossler, 2002; DeRoma et al., 2006; Long \& Sephton, 2011). O sexo, a idade, as habilitações literárias, a profissão, a formação académica, a experiência e conhecimento profissionais têm sido também referidos como variáveis importantes na tomada de decisão (Ben-David, 2011a; Britner \& Mossler, 2002; Cross \& Casanueva, 2009; Davidson-Arad \& Benbenishty, 2008, 2010; Gonçalves, 2007; Herman, 2005; Jent, Eaton, Knickerbocker, Lambert, Merrick, \& Dandes, 2011; Renninger, Veach, \& Bagdale, 2002).

Os fatores situacionais dizem respeito às características da família (maltratante), da criança ou jovem (vítima) e da situação específica de maus-tratos. No que concerne à família, o nível socioeconómico, o emprego precário, a má gestão da casa e a violência são referidos por alguns autores como fatores importantes no momento da tomada de decisão (Andersen \& Fallesen, 2010; Ben-David, 2011a, 2011b; Davidson-Arad, 2001; King, Thatte, \& Trocmé, 2003; White, 2006). Igualmente relevantes são o consumo de substâncias, as doenças físicas ou mentais limitadoras, a monoparentalidade e as relações familiares (e.g., relação entre progenitores e os filhos, entre os primeiros e os companheiros atuais ou anteriores e entre irmãos) (Ben-David, 2011b; Britner \& Mossler, 2002; Christiansen \& Anderssen, 2010; Davidson-Arad, 2001). O relacionamento da família com os serviços sociais, nomeadamente a colaboração, a disponibilidade para a mudança e a responsabilização, é também referido na literatura como um fator 
que influencia a decisão (Arruabarrena \& De Paúl, 2012; Ben-David, 2011b; Davidson-Arad, 2001; Davidson-Arad \& Benbenishty, 2010; DeRoma et al., 2006; Gonçalves, 2007; Littell \& Girvin, 2005; Platt, 2006).

Segundo alguns autores (e.g., Ben-David, 2011b; Britner \& Mossler, 2002; Cross \& Casanueva, 2009; DavidsonArad, 2001; King et al., 2003), existem ainda características da vítima que podem estar relacionadas com os maustratos que lhe são infligidos, tais como a etnia, raça, sexo, idade, nível de desenvolvimento e capacidade de se defender. Estas características parecem afetar os juízos dos técnicos quanto à cronicidade e severidade (Arruabarrena \& De Paúl, 2012; Davidson-Arad \& Benbenishty, 2010; DePanfilis \& Girvin, 2005), mas também relativamente aos indícios desses mesmos maus-tratos (Ashton, 1999; Rossi, Schuerman, \& Budde, 1999). Rossi e colaboradores (1999) consideram que o processo de tomada de decisão relativamente a menores é complexo, frequentemente inconsistente, e marcado por dois tipos de erros: retirada dos menores quando tal não se justifica e manutenção dos menores na família quando se justificaria a retirada.

\section{Realidade Europeia - Particularidades do Sistema Português}

É possível encontrar semelhanças entre os diferentes sistemas judiciais europeus, em particular no que respeita a situações de maus-tratos infantis. Em países como Portugal, Hungria e Suécia os profissionais devem fazer um relatório quando sinalizam um caso de maus-tratos. Na Alemanha e em Portugal, cada profissional (e.g., psicólogos, médicos, assistentes sociais) trabalha sobre diferentes orientações legislativas. Os sistemas alemão, húngaro, sueco, português e holandês possuem uma integração específica dos diferentes serviços disponíveis para a criança e a sua família (Mak \& Steketee, 2012).

Segundo a legislação nacional, com vista a diminuir o risco presente no quotidiano dos menores, é importante que os profissionais ajam de acordo com um conjunto de princípios, estipulados na legislação nacional: superior interesse da criança e do jovem, privacidade e intervenção precoce. É fulcral que a intervenção se inicie no momento em que é conhecida a situação de perigo e não prejudique a criança, protegendo a sua intimidade e vida privada (Lei 147/ 99, 1 de setembro, 1999).

Esta intervenção, e conseguintes decisões, são realizadas apenas por profissionais e instituições especializadas na proteção de menores. Num primeiro nível, encontram-se as entidades com competência em matéria de infância e juventude (ECMIJ), constituídas por diversos profissionais (e.g., psicólogos e assistentes sociais; Lei 147/ 99, 1 de setembro, 1999; Perista \& Silva, 2011). Estas entidades desenvolvem uma ação transversal ao longo de todo o processo, assumindo a coordenação e monitorização do mesmo (Perista \& Silva, 2011).

Quando as EMIJ não conseguem intervir de forma adequada para diminuir o risco de maus-tratos, é necessária a ação das Comissões de Proteção de Crianças e Jovens (CPCJ). Estas, com a autorização dos pais ou do tutor legal, têm poder para decidir e aplicar as medidas de promoção e proteção. Quando a ação das primeiras instâncias não consegue alcançar o objetivo, é necessária a intervenção dos tribunais, que assumem a responsabilidade para com as crianças e jovens em questão (Lei 147/ 99, 1 de setembro, 1999). 


\section{Finalidade do Estudo}

O presente estudo tem como objetivo compreender de que forma grupos de profissionais envolvidos em processos de proteção de menores (psicólogos, professores, educadores sociais e assistentes sociais) ponderam uma eventual retirada das crianças e jovens à família. Especificamente, pretende-se: (a) compreender a opinião dos participantes, tendo em conta a sua profissão e local de trabalho, relativamente aos profissionais envolvidos no processo de tomada de decisão específico de situações de maus-tratos físicos; (b) perceber a opinião dos profissionais, tendo em conta a sua profissão e local de trabalho, no que respeita aos serviços disponibilizados para os menores e para as suas famílias aquando de situações de maus-tratos físicos; (c) analisar a posição de diferentes profissionais, tendo em conta a sua profissão e local de trabalho, face a algumas características de situações de maus-tratos físicos; (d) verificar se a profissão dos participantes e o seu local de trabalho contribuem para o seu processo de tomada de decisão relativamente a medidas de proteção de menores em risco.

Apesar de, a nível internacional, existir uma produção significativa na área dos maus-tratos infantis e de existirem igualmente alguns trabalhos sobre os processos de tomada de decisão por parte dos profissionais, em Portugal existem apenas estudos pontuais sobre esta questão. Não obstante as linhas orientadoras da tomada de decisão serem semelhantes em muitos países, há variações relativas à cultura, valores e princípios de cada sociedade, o que acentua a pertinência do estudo (Long \& Sephton, 2011).

Apesar de em Portugal apenas os juízes deterem o poder legal no que respeita a tomada de decisão face a situações de maus-tratos de menores, existem outros profissionais cujas perceções são relevantes para o desenrolar do processo. Estes profissionais, como por exemplo psicólogos e assistentes sociais, convivem frequentemente com as crianças e as respetivas famílias, pelo que as suas opiniões podem assumir-se como uma mais-valia aquando da tomada de decisão. É ainda de salientar que o modo como estes profissionais percecionam os casos de maus-tratos influencia, em larga escala, o seu modo de agir e a sua intervenção para com as crianças que foram alvo de maustratos e pode influenciar as decisões finais dos magistrados. Neste sentido, é importante compreender como é que estes profissionais - que não têm poder legal mas têm algum poder de facto - formam as suas perceções e ponderam as suas próprias decisões.

\section{MÉTODO}

\section{Participantes}

Participaram neste estudo 68 sujeitos, representando três grupos profissionais envolvidos na proteção de menores: (a) Psicólogos (34\%); (b) Educadores Sociais e Assistentes Sociais (41\%); (c) Professores (25\%). Os participantes eram voluntários, constituindo uma amostra não-probabilística. Os participantes exerciam a sua profissão em quatro locais distintos: 42\% em Comissões de Proteção de Crianças e Jovens (CPCJ), 10\% nas Autarquias, 27\% nas IPSS e $21 \%$ em Escolas. 
A amostra era composta essencialmente por elementos do sexo feminino (97\%), com apenas 3\% de respondentes do sexo masculino. A idade dos participantes variava entre os 24 e os 60 anos e a média de idades era de 35.91 anos $(D P=8.59)$. Oitenta e cinco por cento dos participantes possuíam uma licenciatura, $13 \%$ um mestrado e apenas $2 \%$ eram doutorados.

Existiam diferenças significativas entre os grupos profissionais relativamente à idade, $F(2,65)=12.37, p<.001$. Testes post-hoc de Gabriel revelaram que os Professores eram mais velhos do que os Psicólogos e do que os Educadores Sociais e Assistentes Sociais. Os testes referidos revelaram ainda que os Psicólogos e os Educadores Sociais e Assistentes Sociais não apresentavam diferenças significativas entre si relativamente à idade.

Ao nível das habilitações literárias, o teste de Kruskal-Wallis revelou diferenças significativas entre os diferentes profissionais, $\chi 2(2)=8.09, p=.018$. Testes de Mann-Whitney com correção de Bonferroni $(.05 / 3=.017)$ revelaram que os Educadores Sociais e Assistentes Sociais possuíam menos habilitações literárias que os Psicólogos e os Professores. Os mesmos testes post-hoc mostraram ainda não existirem diferenças significativas entre os Psicólogos e os Professores.

\section{Procedimentos}

Para este estudo a amostra foi recolhida a nível nacional, tendo-se adotado diferentes métodos de abordagem aos participantes. Foi enviado um email de divulgação a dez diretores de instituições de acolhimento de menores e a trinta Comissões de Proteção de Crianças e Jovens. Convidaram-se ainda os alunos de um mestrado da área de promoção e proteção de menores da Universidade do Minho a responder ao questionário, bem como a divulgá-lo pelos seus contactos na área específica de maus-tratos infantis.

\section{Instrumentos}

Os dados foram recolhidos através da colocação na plataforma online "SurveyMonkey" de uma versão adaptada do "Professionals' Decision Making About Out-of-Home Placements" (Britner \& Mossler, 2002). Esta adaptação foi realizada com base na tradução do questionário para a realidade portuguesa tendo em conta o enquadramento legal e os procedimentos do contexto nacional. Antes do instrumento adaptado ser colocado online foi submetido a dois focus groups com profissionais da área.

O questionário é constituído por quatro vinhetas com casos de maus-tratos físicos baseados em factos verídicos e adaptados da realidade portuguesa. Mais especificamente, cada vinheta descreve os maus-tratos físicos a um rapaz (com dois níveis para a idade da criança, 2 e 6 anos) perpetrados pelos progenitores. Foi solicitado que os participantes analisassem cada vinheta individualmente, respondendo a algumas questões.

Pretendeu-se compreender, através de uma questão aberta, quais os serviços que os participantes recomendariam relativamente às situações de maus-tratos (e.g., perícia médico-legal, escola). Procurou-se também analisar a importância atribuída a um conjunto de características específicas de situações de maus-tratos físicos, em que os participantes, a partir de uma escala de tipo Likert de 7 pontos, indicavam a importância de características como, 
por exemplo, a gravidade do abuso, o risco de recorrência e a situação financeira da família. Através de uma lista com diferentes profissionais que, habitualmente, participam no processo de decisão tentou-se verificar que profissionais (e.g., polícia, professor) envolveriam numa eventual tomada de decisão sobre uma medida cautelar.

O questionário integrou ainda uma secção relativa a dados demográficos dos participantes. De seguida apresenta-se uma das vinhetas incluídas no estudo que retrata a situação de uma criança de dois anos:

“O António (nome fictício) é uma criança, de dois anos, proveniente de uma família nuclear (mãe e pai). A referenciação foi realizada por um médico do hospital. A criança tinha contusões nas nádegas e lacerações nas costas, aparentemente provocadas por um cinto ou por um chicote. É a primeira queixa formal à Comissão de Proteção de Crianças e Jovens (CPCJ). A família não teve contactos prévios com os serviços sociais. O horário do pai é variável e raramente está em casa; recusa-se a ser entrevistado pelos profissionais da CPCJ, ordenando-lhes constantemente que se retirem. A mãe aparenta estar deprimida e esgotada; afirma que muitas vezes não consegue lidar com a criança nem com os problemas que esta lhe coloca."

Para controlar potenciais influências de fatores externos, Britner e Mossler (2002) mantiveram constante ao longo de todas as vinhetas o tipo de maus-tratos perpetrados (maus-tratos físicos) e o tipo de família (família nuclear, pai e mãe). Características como a idade da criança (2 ou 6 anos) e o padrão do abuso (abuso crónico ou primeiro abuso) variam, entre as quatro vinhetas, de forma sistemática. De referir que não existiu contrabalanceamento neste estudo, tendo os 4 casos sido apresentados sempre na mesma ordem.

\section{Análise de Dados}

As análises foram realizadas utilizando o Statistical Package for Social Sciences (SPSS, v. 19). Para os testes de significância, foi considerado o critério de $p<.05$.

Num primeiro momento, foi analisada a importância da participação dos diferentes profissionais ao longo do processo. A estatística descritiva permitiu compreender que profissionais são mais relevantes ao longo do processo. Foram ainda utlizados testes de associação (qui-quadrado, $\chi^{2}$ ) para estudar possíveis associações entre os profissionais mais importantes e a profissão e local de trabalho dos participantes.

Devido à estrutura (aberta) da questão relativa aos serviços sugeridos para a criança e a família foi necessário classificar, consoante a natureza do serviço, as respostas dos participantes em três categorias diferentes: serviços de saúde, serviços sociais e de educação, e serviços jurídicos/legais. Para tal, calculou-se o acordo inter-juízes: foram recrutados 8 juízes, membros de um grupo de investigação sobre problemáticas na infância que, de forma independente, classificaram as respostas dos participantes nas três categorias mencionadas.

Utilizou-se novamente a estatística descritiva e os testes de associação. Através da comparação entre grupos (profissão e local de trabalho) estudaram-se possíveis associações relativas aos serviços sugeridos e à profissão e local de trabalho dos participantes. 
Para facilitar as análises, criaram-se 4 grupos de fatores (abuso, criança, família e comunidade) com base na lista de 18 características específicas de situações de maus-tratos apresentadas aos participantes (Figura 2).

\section{FATORES DA FAMÍLIA}

Stress a que os pais estão sujeitos

Psicopatologia dos pais

Resposta dos pais a serviços anteriores

Capacidade cognitiva dos pais

Qualidade das relações das crianças com irmãos ou outros membros da família

Suporte social da família

Abuso de substâncias por parte dos pais

Situação financeira da família

Estabilidade da família

\section{FATORES DA COMUNIDADE}

Disponibilidade de (locais de) acolhimento de qualidade Segurança/ riscos da comunidade envolvente

Serviços que foram disponibilizados

\section{FATORES DA CRIANÇA}

Nível de desenvolvimento da criança

Capacidade da criança recordar o abuso

Vínculo da criança aos pais

\section{FATORES DO ABUSO}

Gravidade do abuso

Risco de recorrência

Duração/ Padrão do abuso

Figura 2. Grupos de características associadas a situações de maus-tratos físicos.

Análises de regressão linear múltipla foram utilizadas para perceber se a profissão e o local de trabalho influenciam a tomada de decisão aquando da atribuição da importância às características de abuso. Para estudar esta relação, foi necessário recodificar as variáveis profissão e local de trabalho através do método effect coding.

\section{RESULTADOS}

\section{Envolvimento de Diferentes Profissionais no Processo de Tomada de Decisão}

Os participantes consideraram que o gestor do processo da CPCJ (88.2\%), os psicólogos clínicos (80.9\%) e os serviços de perícia médico-legal $(79.4 \%)$ são os profissionais mais importantes no desenvolvimento do processo de tomada de decisão. Os tutores legais $(33.8 \%)$ e os polícias $(38.2 \%)$ foram considerados os elementos cuja participação é menos necessária (Figura 3).

Profissão. Num primeiro momento, foram realizados testes de associação qui-quadrado para analisar a associação entre a profissão dos participantes e a importância atribuída ao envolvimento de diferentes profissionais ao longo do processo. Verificou-se que existe uma associação entre a profissão dos participantes e a sua opinião quanto à importância do juiz do Tribunal de Menores ou do Tribunal da Comarca ao longo do processo de tomada de decisão, $\chi^{2}(2)=6.51, p=.04$. Os Educadores Sociais e Assistentes Sociais (71.4\%) e os Professores (70.6\%) consideram importante a colaboração dos Juízes, enquanto que apenas 39.1\% dos Psicólogos da amostra atribuem importância à participação dos Juízes no processo. Relativamente à importância dos outros profissionais, testes qui-quadrado não revelaram associações: tutor legal, $\chi^{2}(2)=3.00, p=.22$; profissional da CPCJ, $\chi^{2}(2)=.65, p=.72$; assistente 
social, $\chi^{2}(2)=.44, p=.80$; serviços de perícia médico-legal, $\chi^{2}(2)=1.51, p=.47$; gestor de processo da CPCJ, $\chi^{2}(2)$ $=3.05, p=.22$; polícia, $\chi^{2}(2)=3.21, p=.11$; psicólogo clínico, $\chi^{2}(2)=3.21, p=.20$; professor, $\chi^{2}(2)=.02, p=.99$.

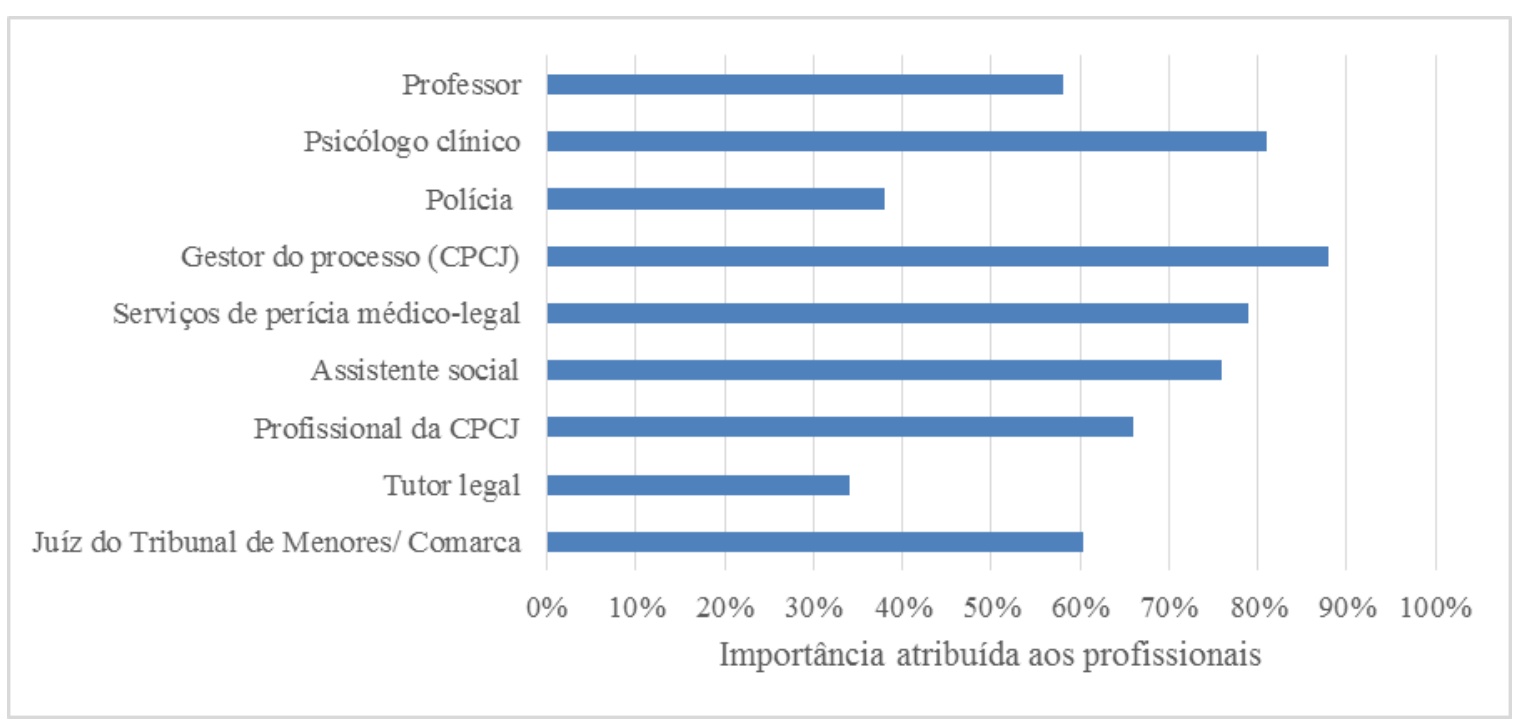

Figura 3. Importância atribuída aos diferentes profissionais.

Local de trabalho. À semelhança do ponto anterior, foi analisada a associação do local de trabalho e da importância da participação de diferentes profissionais no processo. Testes de associação qui-quadrado revelaram que existe uma associação entre a profissão dos participantes e a sua opinião quanto à importância do psicólogo clínico ao longo do processo de tomada de decisão, $\chi^{2}(3)=9.00, p=.03$. Todos os participantes que trabalham em IPSS (100\%) consideram importante a colaboração dos psicólogos clínicos, sendo que a maioria dos participantes que trabalham numa Escola ou numa Autarquia (85\%) e numa CPCJ (65\%) também consideram importante a participação destes profissionais.

Relativamente à importância dos outros profissionais, testes de qui-quadrado não revelaram associações significativas: juiz do Tribunal de Menores ou do Tribunal da Comarca, $\chi^{2}(3)=6.72, p=.08$; tutor legal, $\chi^{2}(3)=$ 2.25, $p=.52$; profissional da CPCJ, $\chi^{2}(3)=3.60, p=.31$; assistente social, $\chi^{2}(3)=2.86, p=.42$; serviços de perícia médico-legal, $\chi^{2}(3)=4.72, p=.19$; gestor de processo da CPCJ, $\chi^{2}(3)=5.41, p=.14$; polícia, $\chi^{2}(3)=.65, p=.09$; professor, $\chi^{2}(3)=5.82, p=.12$.

\section{Serviços Sugeridos pelos Participantes}

Os participantes produziram 72 respostas relativamente aos serviços possíveis, das quais 55 eram diferentes. Estas respostas foram categorizadas de forma independente por oito juízes, tendo em conta três tipos de serviços possíveis (serviços de saúde, e.g., saúde mental; serviços sociais e de educação, e.g., assistência social; e serviços jurídicos/legais, e.g., encaminhamento para o tribunal). De modo a analisar a fiabilidade do acordo na avaliação dos 
diferentes juízes, utilizou-se o kappa de Fleiss $(k)$, tendo-se verificado um elevado acordo $(k=.95)$ para o conjunto de respostas cotadas (Fleiss, 1981).

Os participantes consideram que os Serviços Jurídicos/Legais são os menos importantes no decorrer do processo (com apenas 19.1\% das respostas). Os Serviços de Saúde (45.6\%) foram indicados como os serviços mais relevantes, seguidos dos Serviços Sociais e de Educação (35.3\%).

Profissão. Testes de associação de qui-quadrado mostraram que não existe associação significativa entre a profissão dos participantes e os serviços de saúde, $\chi^{2}(2)=2.61, p=.27$, os serviços sociais e de educação, $\chi^{2}(2)=.79, p=$ .67. No entanto, existe uma associação significativa entre a profissão e a sugestão dos serviços jurídicos/ legais, $\chi^{2}(2)=7.18, p=.04$. Mais especificamente, enquanto que $41 \%$ dos Professores atribuem importância a estes serviços, apenas $13 \%$ e $11 \%$ dos Psicólogos e Educadores Sociais e Assistente Sociais, respetivamente, consideram importantes os serviços da área jurídica. Não foram encontradas associações relativas aos serviços de saúde, $\chi^{2}(2)$ $=2.61, p=.27$, e aos serviços sociais e educativos, $\chi^{2}(2)=.79, p=.67$.

Local de trabalho. Testes de qui-quadrado mostraram que não existe uma associação entre o local de trabalho dos participantes e os serviços sugeridos: serviços de saúde, $\chi^{2}(3)=1.52, p=.68$; serviços sociais e de educação, $\chi^{2}(3)$ $=.93, p=.82$; serviços jurídicos/legais, $\chi^{2}(3)=1.56, p=.67$.

\section{Valoração das Características Específicas em Sitações de Maus-tratos Físicos}

Solicitou-se aos participantes que classificassem 18 características típicas de situações de maus-tratos físicos (e.g., gravidade do abuso, risco de recorrência, situação financeira da família) numa escala de tipo Likert, sendo que 1 correspondia a "Nada importante" e 7 a "Muito Importante". A "gravidade do abuso" $(M=6.85 ; D P=.38)$, o "risco de recorrência" $(M=6.72 ; D P=.65)$ e a "duração/ padrão do abuso" $(M=6.76 ; D P=.59)$ foram consideradas pela generalidade dos participantes como as características dos maus-tratos físicos mais relevantes para uma decisão de retirada de menores à família. No sentido oposto, a "situação financeira da família", foi a menos valorada $(M=4.69$; $D P=1.17$; Quadro 1).

É importante salientar que se observou uma tendência para a atribuição de valores de importância elevados para a maioria das características, sendo que os números 6 e 7 da escala de resposta ("Muito Importante" e "Extremamente Importante") foram selecionados em $65 \%$ das respostas. 
Quadro 1

Características Associadas a Situações de Maus-tratos Infantis

\begin{tabular}{lll} 
Característica & $M$ & $D P$ \\
\hline Gravidade do abuso & 6.85 & 0.38 \\
Risco de recorrência & 6.72 & 0.65 \\
Duração/padrão do abuso & 6.76 & 0.59 \\
Stress a que os pais estão sujeitos & 5.08 & 1.53 \\
Psicopatologia dos pais & 4.95 & 1.50 \\
Resposta dos pais a serviços anteriores & 6.18 & 1.03 \\
Serviços que foram disponibilizados & 5.54 & 1.37 \\
Capacidade cognitiva dos pais & 6.27 & 0.99 \\
Qualidade das relações das crianças com irmãos ou outros membros da & 5.84 & 1.12 \\
família & & \\
Nível de desenvolvimento da criança & 5.13 & 1.66 \\
Disponibilidade de (locais de) acolhimento de qualidade & 5.79 & 1.20 \\
Capacidade da criança recordar o abuso & 5.44 & 1.53 \\
Segurança/riscos da comunidade envolvente & 5.35 & 1.22 \\
Suporte social da família & 5.90 & 1.08 \\
Estabilidade da família & 5.52 & 1.38 \\
Abuso de substâncias por parte dos pais & 5.70 & 1.52 \\
Vínculo da criança aos pais & 5.42 & 1.61 \\
Situação financeira da família & 4.69 & 1.17 \\
\hline Nota. A escala de resposta varia entre 1 (“Nada Importante”) e 7 (“Extremamente Importante”) \\
\hline
\end{tabular}

Com vista a possibilitar a realização das análises seguintes, as 18 características foram agrupadas em 4 categorias abuso, criança, família e comunidade (ver Figura 2).

Profissão. Testes de Kruskal-Wallis que não relevaram diferenças significativas no modo como participantes com profissões diferentes avaliam os fatores considerados: fatores do abuso, $H(2)=5.43, p=.07$; fatores da comunidade, $H(2)=4.70, p=.10$; fatores da criança, $H(2)=1.70, p=.43$; fatores da família, $H(2)=2.10, p=$ .35 .

Local de trabalho. Também não foram encontradas diferenças no modo como profissionais atuando em contextos diversos (e.g., (Autarquia, CPCJ, IPSS, Escola) avaliam os fatores específicos de situações de maus-tratos: fatores da comunidade, $H(3)=6.84, p=.77$; fatores da criança, $H(3)=2.68, p=.44$; fatores da família, $H(2)=6.21$, $p=.10$, com exceção dos fatores relativos ao abuso, $H(3)=11.68, p=.01$.

Os testes post-hoc de Mann-Whitney com correção de Bonferroni $(p<.008)$, revelaram apenas diferenças entre participantes que trabalham em IPSS e participantes que trabalham nas CPCJ $(U=125.00, p=.01)$. Mais especificamente, quem trabalha nas CPCJ considera os fatores caracterizadores dos maus-tratos físicos como menos 
importantes do que os profissionais que exercem em IPSS.

\section{Preditores das Características Específicas em Sitações de Maus-tratos Físicos}

Com vista a compreender um possível efeito das variáveis profissão e local de trabalho na tomada de decisão relativamente à lista de características, foi conduzida uma análise de regressão múltipla. Inicialmente, foi levada a cabo uma recodificação das variáveis profissão e local de trabalho com vista ser possível realizar a análise proposta. Para tal, foi utilizado o método effect coding.

Foram testados quatro modelos de regressão linear múltipla, um para cada um dos grupos de características de maustratos. Os modelos que consideraram individualmente os fatores da comunidade, $R^{2}(\mathrm{Aj})=.64, F(5,62)=1.92, p=$ .104 , os fatores da criança, $R^{2}(\mathrm{Aj})=.01, F(5,62)=1.02, p=.41$, e os fatores da família, $R^{2}(\mathrm{Aj})=.68, F(5,62)=$ $1.98, p=.09$, não se revelaram explicativos dos dados recolhidos.

Apenas o modelo de regressão que inclui os fatores caracterizadores do abuso é estatisticamente significativo, $R^{2}(\mathrm{Aj})$ $=.11, F(5,62)=2.63, p=.03$, explicando $17.5 \%$ da variância da tomada de decisão. A profissão e o local de trabalho revelaram-se preditores significativos da importância atribuída aos fatores caracterizadores do abuso, sendo que os profissionais das autarquias atribuem uma importância reduzida aos fatores referidos, $\beta=-.35, t=-2.34, p=.02$. Por outro lado, os professores atribuem muita importância aos fatores mencionados aquando da tomada de decisão, $\beta=.30, t=2.01, p=.05$.

\section{DISCUSSÃO}

O presente estudo abrange a tomada de decisão de profissionais em situações específicas de maus-tratos infantis. Foram estudadas as perceções dos participantes face aos serviços disponibilizados para a criança e para a família e também sobre a importância do envolvimento de determinados profissionais no processo. Por serem um constructo tão complexo, os maus-tratos infantis possuem vários fatores que lhes são característicos. Também foram estudadas as opiniões dos profissionais face aos fatores específicos destas situações.

O facto de, no estudo, $65 \%$ das características dos maus-tratos serem consideradas como "extremamente importantes" ou "muito importantes" parece evidenciar a complexidade do processo de tomada de decisão na retirada de menores à família, já referenciada por estudos anteriores (e.g., Britner e Mossler, 2002; Cleaver \& Walker, 2004). Para além da perceção da complexidade da tarefa, os participantes poderão igualmente considerar a tarefa como de elevada responsabilidade, pelo que tenderão a considerar como indispensável a cuidadosa ponderação de múltiplos fatores, procurando assim diminuir a margem de erro.

Por outro lado, e ao contrário dos resultados encontrados por outros autores (Andersen \& Fallesen, 2010; Ben-David, 2011a, 2011b; Davidson-Arad, 2001; King et al., 2003; White, 2006), os participantes no estudo não consideraram o nível socioeconómico (NSE), considerado como situação financeira da família, como um fator relevante para a tomada de decisão. A desejabilidade social e a necessidade de os participantes se demarcarem de qualquer opção 
que pudesse ser encarada como discriminatória, poderão ser algumas das razões que conduziram a este resultado inesperado. Por outro lado, se é verdade que as situações de maus-tratos são transversais a todos os estratos sociais, a grande maioria dos menores acolhidos provém dos estratos sociais mais desfavorecidos (Alberto, 2002). Eventualmente, os participantes ajuízam que maltrato é sempre maltrato, independentemente do NSE, apesar de este ser, generalizadamente, considerado um fator de risco para a continuação dos maus-tratos.

Outro resultado importante prende-se com a relevância atribuída pelos participantes ao risco de recorrência do abuso. A impossibilidade de prever um futuro maltrato é referida na literatura como um fator que influencia em larga escala a tomada de decisão, tornando-a complexa e suscetível a enviesamentos (e.g., Benbenishty et al., 2003; Gambrill, 2005). Porventura, existirão estratégias (não exploradas neste estudo) utilizadas pelos profissionais para predizer a ocorrência (ou não) de maus-tratos futuros. Independentemente do sentido em que os participantes relevam ou perspetivam a possibilidade de recorrência do abuso, os resultados sugerem que quer a recorrência comprovada quer a possível recorrência de abusos constituem fatores percecionados como muito relevantes para a tomada de decisão de retirada dos menores.

A classificação, por parte dos participantes, da gravidade, padrão e duração do abuso como características classificadas "muito importantes" é consistente com outros estudos (Crea, 2010; Cross \& Casanueva, 2009; Molina, 2010; Trocmé, Knoke, Fallon, \& MacLaurin, 2009). Aparentemente, quando os maus-tratos infantis são percecionados com um nível de severidade elevado, é mais provável que a criança seja retirada à família (DeRoma, Bingley, Kessler, \& Merino, 2005). O padrão e duração do abuso também é, aparentemente, uma característica com um impacto significativo na decisão dos profissionais (Britner \& Mossler, 2002; DePanfilis \& Girvin, 2005; Dettlaff, Rivaux, Baumann, Fluke, Rycraft, \& James, 2011).

É ainda importante destacar a diferença entre os participantes que exercem numa CPCJ e numa IPSS, ao nível das características dos maus-tratos. A literatura não aponta nenhuma explicação para o facto de os participantes que exercem numa CPCJ atribuírem menos importância aos fatores dos maus-tratos do que aqueles que trabalham numa IPSS. Eventualmente, o contexto de trabalho poderá pesar no momento da tomada de decisão, já que os profissionais tenderão a apoiar-se em experiências e casos com os quais contactam no seu contexto laboral.

No que respeita à profissão e ao local de trabalho dos participantes, quando considerados em conjunto (estudo preditivo), são indicadores que parecem explicar uma parte da tomada de decisão, em particular no que diz respeito aos fatores do abuso (risco de recorrência, duração/padrão e gravidade do abuso). Este resultado corrobora conclusões de outros estudos (e.g., Lopes \& Fernandes, 2009) que têm evidenciado que o tipo de formação inicial é relevante para as práticas profissionais mas que o contexto tem igualmente algum impacto nestas práticas. É provável que a maior ou menor ponderação comparada destes fatores seja muito específica das situações em estudo, o que justificará que, no caso particular do estudo, a decisão sobre os fatores de maus-tratos seja, em parte, explicada pela profissão e local de trabalho dos participantes e nas restantes categorias os indicadores não sejam significativos.

Os resultados evidenciam ainda que existe consistência entre os serviços recomendados e os profissionais considerados como necessários ao desenrolar do processo. Os participantes referenciaram os serviços de saúde como 
os mais importantes nas situações de maus-tratos, bem como os profissionais de perícia médico-legal e os psicólogos. Estes resultados são idênticos aos encontrados por outros autores (e.g., Britner e Mossler, 2002). De salientar o facto de os psicólogos atribuírem uma importância reduzida aos serviços jurídicos e legais, o que pode dever-se em parte ao método de constituição (não aleatória) da amostra, que não garantiu que todos os participantes da área de psicologia tivessem experiência em situações de maus-tratos.

Os tutores legais e os polícias foram considerados como os profissionais menos indispensáveis ao processo de tomada de decisão. Os profissionais de segurança pública, embora tenham um papel crucial na articulação e no reconhecimento de situações problemáticas, agem em geral em momentos pontuais e essencialmente na fase inicial do processo (Magalhães, 2005), o que poderá contribuir para a relativização da sua importância face a outros profissionais. Dependendo do grau de conhecimento sobre o processo conduzido por uma equipa multidisciplinar, os profissionais podem encarar os polícias como aqueles que se limitam a repor a ordem a mando de terceiros e cujo papel poderá ser, por isso mesmo, menos valorizado.

No que diz respeito aos tutores legais, modelos recentes sobre a tomada de decisão em equipa defendem a participação da família (tutor legal) na tomada de decisão com vista a fortalecer as relações familiares e a aumentar a estabilidade e o apoio emocional dos progenitores (Abramson-Madden, Crampton, Crea, \& Usher, 2008; Connolly \& Morris, 2012; Davidson-Arad \& Benbenishty, 2008; Gunderson, Cahn, \& Wirth, 2003). Os participantes não parecem, contudo, adotar esta perspetiva, eventualmente porque o modelo instituído em Portugal, em que a família nem sempre assume um papel ativo no processo, apresenta características diferentes das de outros países.

De salientar a importância de que o gestor do processo da CJCP aparentemente se reveste, tendo sido indicado por 88.2\% dos participantes como o profissional mais importante ao longo do processo. Com responsabilidades como a recolha e gestão de informações, realização de diligências e monitorização da intervenção, o seu acompanhamento ao longo de todo o processo é essencial (Repensar Intervenção Social, 2012). No entanto, apesar do seu papel ativo (Silva, 2009), a sua participação não tem sido alvo de muitos estudos.

\section{Limitações}

A generalização dos resultados encontrados neste estudo está limitada pela amostra e metodologia utilizadas. A opção de colocar o questionário numa plataforma online acarretou algumas consequências, particularmente no que respeita ao preenchimento completo do mesmo, tendo-se verificado um decréscimo acentuado de respostas ao longo do questionário. No que respeita aos participantes, a variedade de profissões ficou um pouco aquém das expectativas.

A validade dos resultados pode ainda ser afetada pela utilização de vinhetas com casos hipotéticos, a qual implica um distanciamento face à realidade (Portwood, 1998), uma vez que a informação disponibilizada não inclui toda a informação a que os profissionais podem ter acesso numa situação real (Ibanez et al., 2006; Jent et al., 2011). Deste modo, os resultados terão que ser interpretados com cautela. Por outro lado, as situações apresentadas aos participantes retratam apenas casos de maus-tratos físicos, pelo que qualquer conclusão terá que ficar limitada a este tipo de maus-tratos. Além disso, a amostra utilizada pode não representar a realidade nacional específica de 
profissionais que se dedicam a este tipo de situações. Na verdade, não está garantido que todos os profissionais constituintes da amostra possuam experiência em situações de maus-tratos-infantis. Deste modo, os resultados podem estar, em parte, enviesados, não representando um retrato fidedigno da realidade.

Por fim, a apresentação dos casos em vinhetas não seguiu o modelo adotado por Britner e Mossler (2002), os quais apresentam os casos aleatoriamente, eliminando deste modo o fator do cansaço do participante. Neste estudo, a ordem de apresentação dos casos foi sempre a mesma, não existindo contrabalanceamento.

\section{Implicações Práticas e Estudos Futuros}

A nível prático, a pertinência do estudo prende-se com a possibilidade de, através de uma melhor compreensão do processo, facilitar a tomada de decisão. A agilização destes processos de promoção e proteção e consequente decisão é crucial para o seu sucesso. Compreender de que modo os profissionais de primeira linha perspetivam as situações de maus-tratos permite facilitar a interação entre entidades e famílias. Com os recursos geralmente insuficientes e o tempo sempre escasso, urge intervir de forma objetiva e direcionada, o que só é possível se tivermos conhecimento do desenrolar do processo.

A inclusão de profissionais da área de justiça neste tipo de estudos é uma mais-valia. Os tribunais, e especificamente os juízes, detêm o poder legal sobre os processos de promoção e proteção de menores, sendo, em último recurso, quem se responsabiliza pelas crianças e jovens (Lei 147/99, 1 de setembro, 1999). Neste sentido, e por se constituírem como uma categoria profissional importante no processo de tomada de decisão, seria interessante perceber qual a posição dos juízes face a casos de maus-tratos.

Seria igualmente interessante compreender de que modo fatores demográficos como o sexo e as habilitações literárias podem afetar estas tomadas de decisão. A reduzida variabilidade da amostra do presente estudo não permitiu averiguar este tipo de relação. No entanto, será importante em futuras investigações abordar este tipo de variáveis.

As características que foram estudadas no presente estudo foram baseadas no estudo de Britner e Mossler (2002), ou seja, são características baseadas em investigação. Será que na realidade nacional também as características aqui utilizadas serão as mais frequentes? Através, por exemplo, da consulta de processos, poderá ser possível compreender quais os fatores das situações de maus-tratos em que se baseia a decisão real.

\section{REFERÊNCIAS}

Abramson-Madden, A., Crampton, D. S., Crea, T. M., \& Usher, C. L. (2008). Variability in the implementation of team decisionmaking (TDM): Scope and compliance with the family to family practice model. Children and Youth Services Review, 30, 1221-1232. doi:10.1016/j.childyouth.2008.03.007 
Alberto, I. M. (2002). Como pássaros em gaiolas? Reflexões em torno da institucionalização de menores em risco. In C. Machado \& R. A. Gonçalves (Eds.), Violência e vítimas de crimes - Crianças (pp. 13-36). Coimbra: Quarteto Editora.

Andersen, S. H., \& Fallesen, P. (2010). A question on class: On the heterogeneous relationship between background characteristics and a child's placement risk. Children and Youth Service Review, 32(6), 783-789.

Arruabarrena, I., \& De Paúl, J. (2012). Improving accuracy and consistency in child maltreatment severity assessment in child protection services in Spain: New set of criteria to help caseworkers in substantiation decisions. Children and Youth Services Review, 34, 666-674. doi:10.1016/j.childyouth.2011.12.011

Ashton, V. (1999). Worker judgements of seriousness about and reporting of suspected child maltreatment. Child Abuse \& Neglect, 23(6), 539-548.

Ashton, V. (2001). The relationship between attitudes toward corporal punishment and the perception and reporting of child maltreatment. Child Abuse \& Neglect, 25, 389-399.

Baird, C., \& Wagner, D. (2000). The relative validity of actuarial- and consensus-based risk assessment systems. Children and Youth Services Review, 22(11-12), 839-871. doi:10.1016/S0190-7409(00)00122-5

Ben-David, V. (2011a). Judicial bias in adjudicating the adoption of minors in Israel. Children and Youth Services Review, 33, 195-203. doi:10.1016/j.childyouth.2010.09.003

Ben-David, V. (2011b). Social information in court decisions of compulsory child adoption in Israel. Child \& Youth Care Forum, 40, 233-249. doi:10.1007/s10566-010-9133-3

Benbenishty, R., Osmo, R., \& Gold, N. (2003). Rationales provided for risk assessments and for recommended interventions in child protection: A comparison between Canadian and Israeli professionals. British Journal of Social Work, 33, 137155.

Repensar Intervenção Social. (2012). Boas Práticas na Intervenção Social. Retrieved from www.ua.pt/ris/ReadObject.aspx?obj=25386

Britner, P., \& Mossler, D. (2002). Professionals' decision-making about out-of-home placements following instances of child abuse. Child Abuse \& Neglect, 26(4), 317-32. Retrieved from http://www.ncbi.nlm.nih.gov/pubmed/12092801

Brofenbrenner, U. (1977). Toward an experimental ecology of human development. American Psychologist, 32(7), 513-531. doi:10.1037/0003-066X.32.7.513

Christiansen, O., \& Anderssen, N. (2010). From concerned to convinced: Reaching decisions about out-of-home care in Norwegian Child Welfare Services. Child \& Family Social Work, 15, 31-40. doi:10.1111/j.1365-2206.2009.00635.x

Cleaver, H., \& Walker, S. (2004). From policy to practice: The implementation of a new framework for social work assessments of children and families. Child \& Family Social Work, 9, 81-90.

Comissão Nacional de Protecção das Crianças e Jovens em Risco. (2011). Promoção e protecção dos direitos das crianças: Guia de orientações para os profissionais da acção social na abordagem ade situações de maus tratos ou outras situações de perigo. Lisboa. Retrieved from http://www.cnpcjr.pt/downloads/Guia_accao_social.pdf

Connolly, M., \& Morris, K. (2012). Family decision making in child welfare: Challenges in developing a knowledge base for practice. Child Abuse Review, 21(1), 41-52. doi:10.1002/car.1143

Crea, T. M. (2010). Balanced decision making in child welfare: Structured processes informed by multiple perspectives. Administration in Social Work, 34, 196-212. doi:10.1080/03643101003609529

Cross, T. P., \& Casanueva, C. (2009). Caseworkers judgments and substantiation. Child Maltreatment, 14, 38-52.

Davidson-Arad, B. (2001). Parental features and quality of life in the decision to remove children at risk from home. Child Abuse \& Neglect, 25, 47-64. Retrieved from http://www.ncbi.nlm.nih.gov/pubmed/11214812

Davidson-Arad, B., \& Benbenishty, R. (2008). The role of workers' attitudes and parent and child wishes in child protection 
workers' assessments and recommendation regarding removal and reunification. Children and Youth Services Review, 30, 107-121. doi:10.1016/j.childyouth.2007.07.003

Davidson-Arad, B., \& Benbenishty, R. (2010). Contribution of child protection workers' attitudes to their risk assessments and intervention recommendations: A study in Israel. Health \& Social Care in the Community, 18(1), 1-9. doi:10.1111/j.1365-2524.2009.00868.x

Davidson-Arad, B., Englechin-Segal, D., \& Wozner, Y. (2003). Short-term follow-up of children at risk: Comparison of the quality of life of children removed from home and children remaining at home. Child Abuse \& Neglect, 27(7), 733750. doi:10.1016/S0145-2134(03)00113-3

DePanfilis, D., \& Girvin, H. (2005). Investigating child maltreatment in out-of-home care: Barriers to effective decisionmaking. Children and Youth Services Review, 27, 353-374. doi:10.1016/j.childyouth.2004.11.010

Department of Health, Department of Education and Employment Home Office. (2000). Framework for the assessment of children in need and their families. London: TSO [The Stationery Office]

DeRoma, V., Bingley, C., Kessler, M. L., \& Merino, C. (2005). Unacceptable risk factors in child maltreatment: Formulations from caseworkers. Internacional Journal of Behavioral Consultation and Therapy, 1(2), 114-123.

DeRoma, V. M., Kessler, M. L., McDaniel, R., \& Soto, C. M. (2006). Important risk factors in home-removal decisions: Social caseworker perceptions. Child and Adolescent Social Work Journal, 23(3), 263-277. doi:10.1007/s10560-006-00478

Dettlaff, A. J., Rivaux, S. L., Baumann, D. J., Fluke, J. D., Rycraft, J. R., \& James, J. (2011). Disentangling substantiation: The influence of race, income, and risk on the substantiation decision in child welfare. Children and Youth Services Review, 33, 1630-1637. doi:10.1016/j.childyouth.2011.04.005

Drury-Hudson, J. (1999). Decision-making in child protection: The use of theoretical, empirical and procedural knowledge by novices and experts and implications for fieldwork placement. British Journal of Social Work, 29, 147-169.

English, D. J., Marshall, D. B., Coghlan, L., Brummel, S., \& Orme, M. (2002). Causes and consequences of the substantiaion decision in Washington State child protective services. Children and Youth Services Review, 24(11), 817-851.

Everson, M. D., \& Sandoval, J. M. (2011). Forensic child sexual abuse evaluations: Assessing subjectivity and bias in professional judgements. Child Abuse \& Neglect, 35, 287-298. doi:10.1016/j.chiabu.2011.01.001

Gambrill, E. D. (2005). Decision making in child welfare: Errors and their context. Children and Youth Services Review, 27, 347-352. doi:10.1016/j.childyouth.2004.12.005

Gambrill, E. D. (2011). Evidence-based practice and the ethics of discretion. Journal of Social Work, 11, $26-48$.

Gonçalves, C. (2007). Avaliação e intervenção com famílias multiproblemáticas: Um olhar sobre os profissionais. Faculdade de Psicologia e de Ciências da Educação da Universidade de Coimbra, Coimbra.

Gunderson, K., Cahn, K., \& Wirth, J. (2003). The Washington State long-term outcome study. Protecting Children, 18(1), 4247.

Herman, S. (2005). Improving decision making in forensic child sexual abuse evaluations. Law and Human Behavior, 29(1), 87-120. doi:10.1007/s10979-005-1400-8

Ibanez, E. S., Borrego, J., Pemberton, J. R., \& Terao, S. (2006). Cultural factors in decision-making about child physical abuse: Identifying reporter characteristics influencing reporting tendencies. Child Abuse \& Neglect, 30, $1365-79$. doi:10.1016/j.chiabu.2006.06.007

Jent, J. F., Eaton, C. K., Knickerbocker, L., Lambert, W. F., Merrick, M. T., \& Dandes, S. K. (2011). Multidisciplinary child protection decision making about physical abuse: Determining substantiation thresholds and biases. Children and Youth Services Review, 33, 1673-1682. doi:10.1016/j.childyouth.2011.04.029

King, G., Thatte, N., \& Trocmé, N. (2003). Substantiation as a multitier process: The results of a NIS-3 analysis. Child 
Maltreatment, 8(3), 173-182.

Lei 147/ 99, 1 de setembro, Pub. L. No. Diário da República (1999). Portugal. Retrieved from http://www.pgdlisboa.pt/

Littell, J. H., \& Girvin, H. (2005). Caregivers' readiness for change: Predictive validity in a child welfare sample. Child Abuse \& Neglect, 29, 59-80. doi:10.1016/j.chiabu.2004.08.004

Long, M., \& Sephton, R. (2011). Rethinking the "best interests" of the child: Voices from aboriginal child and family welfare practitioners. Australian Social Work, 64(1), 96-112. doi:10.1080/0312407X.2010.535544

Lopes, J., \& Fernandes, P. (2009). Emergent literacy beliefs in preschool and kindergarten contexts. L1 - Educational Studies in Language and Literature, 9, 5-26.

Magalhães, T. (2005). Maus tratos em crianças e jovens. Coimbra: Quarteto Editora.

Mak, J., \& Steketee, M. (2012). Prevent and combat child abuse and neglect. Retrieved from http://www.verweyjonker.nl/doc/jeugd/Internationaal-Prevent-and-Combat-Child-Abuse-and-Neglect_7254_web.pdf

Molina, A. (2010). La toma de decisiones profesionales en Protección de Menores. Retrieved from http://www.fapmi.es/imagenes/ subsecciones1/MT_01_B_Antonio\%20Molina\%20Facio_PONEN CIA_web.pdf.

Munro, E. (2010). Learning to reduce risk in child protection. British Journal of Social Work, 40, 1135-1151.

Perista, P., \& Silva, M. (2011). Combating child abuse and neglect in Portugal. http://www.kenniscentrum-kjp.nl

Platt, D. (2006). Threshold decisions: How social workers prioritize referrals of child concern. Child Abuse Review, 15(1), 418. doi:10.1002/car.929

Portwood, S. G. (1998). The impact of individuals' characteristics and experiences on their definitions of child maltreatment. Child Abuse \& Neglect, 22(5), 437-452.

Renninger, S. M., Veach, P. M., \& Bagdale, P. (2002). Psychologists' knowledge, opinions, and decision-making processes regarding child abuse and neglect reporting laws. Professional Psychology Research and Practice, 33(1), 19-23.

Rossi, P. H., Schuerman, J., \& Budde, S. (1999). Understanding decisions about child maltreatment. Evaluation Review, 23(6), 579-598.

Schwalbe, C. S. (2004). Re-visioning risk assessment for human service decision making. Children and Youth Services Review, 26(6), 561-573.

Silva, A. (2009). Discursos sobre o papel do Professor na CPCJ. Covilhã, Portugal Universidade da Beira Interior.

Slep, A. M. S., \& Heyman, R. E. (2006). Creating and field-testing child maltreatment definitions: Improving the reliability of substantiation determinations. Child Maltreatment, 11, 397-408.

Trocmé, N., Knoke, D., Fallon, B., \& MacLaurin, B. (2009). Differentiating between substantiated, suspected, and unsubstantiated maltreatment in Canada. Child Maltreatment, 14, 4-16.

Wells, S. J., Lyons, P., Doueck, H. J., Brown, C. H., \& Thomas, J. (2004). Ecological factors and screening in child protective services. Children and Youth Services Review, 26, 981-997. doi:10.1016/j.childyouth.2004.05.002

White, C. (2006). Federally mandated destruction of the black family: The adoption and safe family act. Northwestern Journal of Law and Social Policy, 1(1), 303-337.

Yoo, J., \& Brooks, D. (2005). The role of organizational variables in predicting service effectiveness: An analysis of a multilevel model. Research on Social Work Practice, 15, 267-277. 